\title{
Human Embryoid Bodies Containing Nano- and Microparticulate Delivery Vehicles**
}

\author{
By Lino Ferreira, Trevor Squier, Hyoungshin Park, Hannah Choe, Daniel S. Kohane, and \\ Robert Langer*
}

During normal embryogenesis, human embryonic stem cells (hESCs) differentiate along different lineages in the context of complex 3D tissue structures where various growth factors direct the differentiation process at different times. Embryoid bodies (EBs) are frequently used as a means to achieve specific stages of embryogenesis in vitro. ${ }^{[1-5]}$ They can be formed from hESC aggregates removed from a feeder layer and cultured in suspension (termed regular EBs). EBs allow cell-cell interaction and they can be rapidly expanded to yield differentiated cells by the use of bioreactors. ${ }^{[6,7]}$ Because of heterogeneous size and spontaneous differentiation of EBs into the three germ layers of the embryo including ectoderm, mesoderm, and endoderm, ${ }^{[1]}$ it is generally difficult to control their differentiation. Specifically for vascular differentiation, between 2 and $10 \%$ of cells in EBs can be isolated as endothelial cells or endothelial progenitor cells after 10-13 days of differentiation. $^{[2,3]}$ Strategies to control the vascular differentiation of EBs could help increase the yield of vascular cells, while contributing to a better understanding of the different growth factors involved in this process.

We hypothesize that the incorporation of particulate growthfactor-delivery vehicles in EBs will enhance their vascular

[*] Prof. R. Langer, L. Ferreira, T. Squier, H. Park, H. Choe, D. S. Kohane

Department of Chemical Engineering

Massachusetts Institute of Technology

E25-342 MIT, 77 Massachusetts Avenue

Cambridge, MA 02139 (USA)

E-mail: rlanger@mit.edu

L. Ferreira

Center of Neurosciences and Cell Biology

University of Coimbra

3004-517 Coimbra (Portugal)

L. Ferreira

Biocant - Biotechnology Innovation Center

3060-197 Cantanhede (Portugal)

D. S. Kohane

Laboratory for Biomaterials and Drug Delivery

Department of Anesthesia

Division of Critical Care Medicine

Children's Hospital Boston

300 Longwood Ave., Boston, MA 02115 (USA)

[**] The authors would like to thank Jason Fuller and Jon Aanestad for valuable suggestions and the financial support of Fundação para a Ciência e a Tecnologia (SFRH/BPD/14502/2003; fellowship to L.F), NIH (grants HL060435, DE13023 and HL076485) and the MIT-Portugal Program (focus in Bioengineering). Supporting Information is available online from Wiley InterScience or from the authors. differentiation by increasing the growth factor concentration within the EBs, potentially at doses and in time frames determined by the method of manufacture of both the particles and EBs. In addition, the local delivery of growth factors within these 3D cellular structures will likely extend the duration of exposure of cells to the growth factors, which otherwise tend to have short half-lives. ${ }^{[8]}$ Previously, nerve-growth-factorreleasing particles assembled with fetal brain cells have been reported to enhance cell survival and functionality; however, the role of these particles in cell differentiation was unclear. ${ }^{[9]}$ Another advantage of incorporating particles with EBs is that it ensures that the cells and the drug delivery system will be implanted together and subsequently stay together after placement in vivo.

Here we present a novel methodology to enhance the vascular differentiation of hESCs by the incorporation of growth-factor-releasing particles in EBs. We demonstrate that the incorporation of these particles has a minimal effect on cell viability and proliferation but a large impact on differentiation. In some cases, the effect on vascular differentiation of particles containing growth factors was superior to that observed by exposing EBs to large extrinsic doses of the same growth factors. In addition, we studied the intracellular trafficking of particles of different sizes within hESCs. We further demonstrate that nanoparticles can be taken up by hESCs and will accumulate in the perinuclear region.

To form EBs with a defined size we deposited between 15000 and $60000 \mathrm{hESCs}$ in round-bottomed, low-adherence, 96 well plates and induced aggregation by centrifugation. ${ }^{[10]} \mathrm{EBs}$ with diameters between 340 and $820 \mu \mathrm{m}$ were obtained by changing the initial seeding number of cells (Supporting Information, Fig. S1). EBs formed by 15000 or 30000 cells showed no significant changes in metabolic activity as measured by a (3-[4,5-dimethylthiazol-2-yl]-2,5-diphenyl-tetrazolium bromide (MTT) assay for 10 days while a decrease was observed for EBs formed by a large number of cells (above 30000; Supporting Information Fig. S1). Based on these results, we used 30000 cells for subsequent experiments and the resulting EBs differentiated in suspension for 10 days based on previous results in the differentiation of hESCs into the vascular lineage. ${ }^{[2]}$

We used biocompatible and biodegradable poly(lactideco-glycolide) (PLGA) particles with different sizes as a model system to design growth-factor-releasing systems (Fig. 1A and Supporting Information Table S1). We evaluated the morphology and degradation of these particles at physiologic $\mathrm{pH}$ 


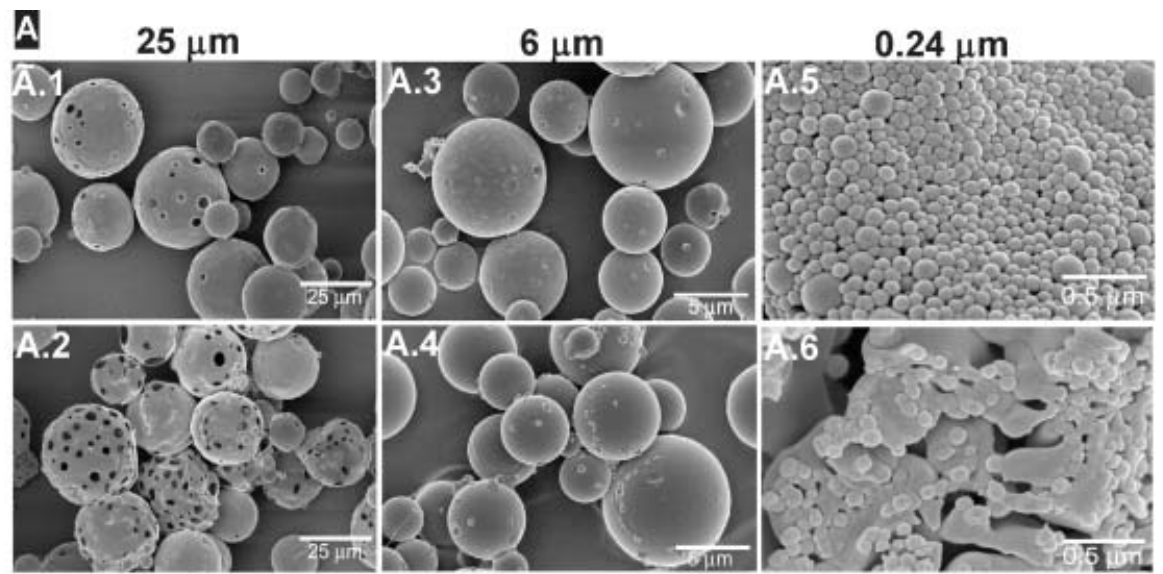

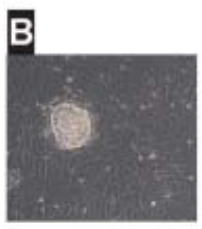

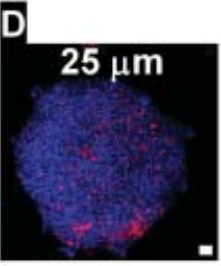

Bottom
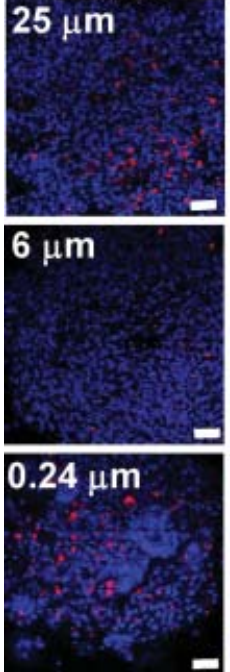

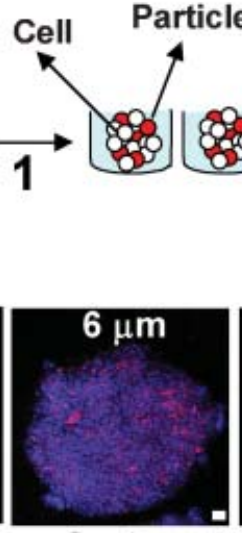

Center
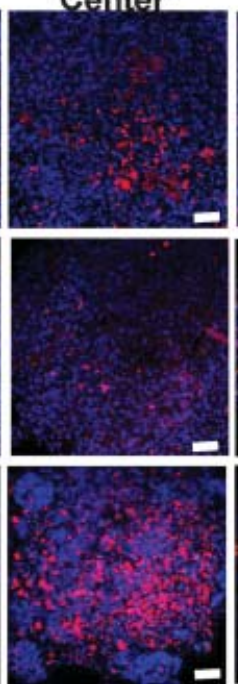

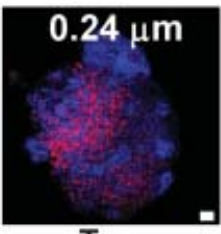

Top
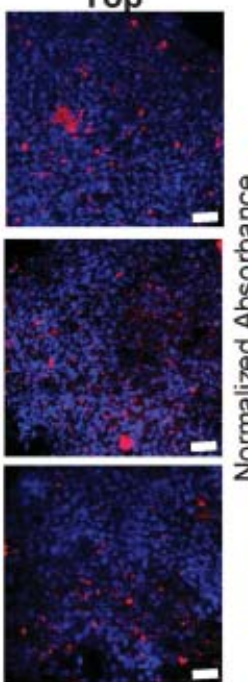

Immunocytochemistry

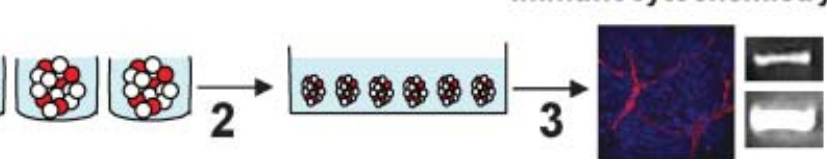

RT-PCR
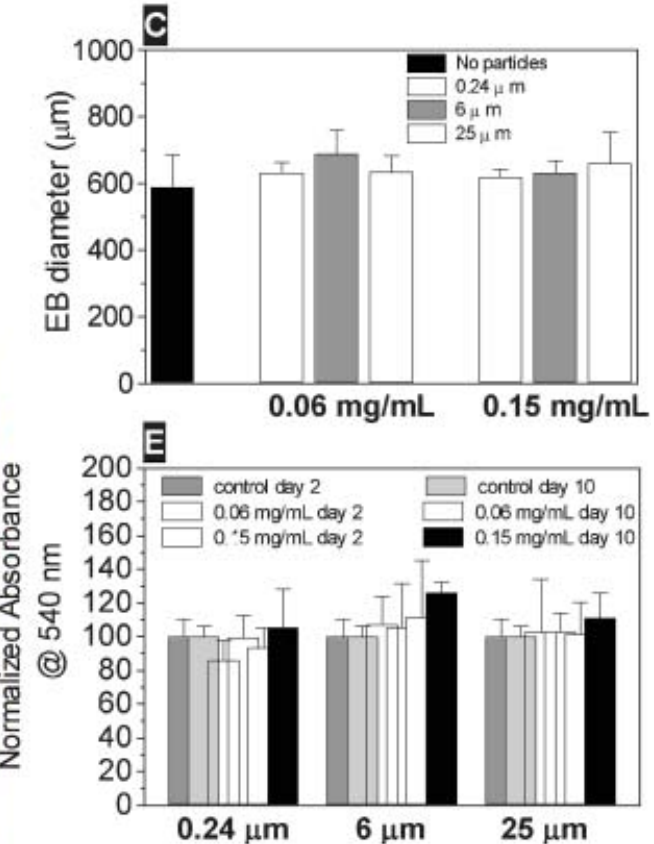

Figure 1. Preparation and viability of EBs containing particles. A) Scanning electron microscopy of particles $25 \mu \mathrm{m}$ (A.1, A.2), $6 \mu \mathrm{m}$ (A.3, A.4), and $0.24 \mu \mathrm{m}$ (A.5, A.6) at day 0 (A. 1, A.3, A.5) and 10 (A.2, A.4, A.6), after incubation in PBS at $37^{\circ}$ C. B) Scheme for the formation of EBs containing particles. 1) Undifferentiated hESC colonies were dissociated into single cells which were aggregated with particles of different sizes in a 96 well-plate by centrifugation forming EBs. 2) After 2 days, EBs were transferred to a low adhesion 24-well plate and cultured for additional 8 days in differentiation medium. 3) EBs were then characterized by immunocytochemistry and quantitative RT-PCR. C) Size of EBs (average \pm S.D., $n=13$ ) formed by the aggregation of 30000 cells in the presence or absence of different concentrations of PLGA particles. D) Distribution of TRITC-labeled particles with different sizes in EBs at different depths, as evaluated by confocal microscopy. EBs were formed by the aggregation of $30000 \mathrm{hESCs}$ and $0.15 \mathrm{mg} \mathrm{mL} \mathrm{m}^{-1}$ of particles. Bar corresponds to $100 \mu \mathrm{m}$. E) Mitochondrial metabolic activity (average \pm S.D., $n=6$ ) of EBs formed by the aggregation of 30000 hESCs and different concentrations of particles with different size, at day 2 and 10 . The absorbances at $540 \mathrm{~nm}$ were normalized by control day 2 absorbance. 
(in PBS, pH 7.4) since this parameter will affect growth factor release. After 10 days in PBS, small-size microparticles $(6 \mu \mathrm{m})$ kept their initial surface morphology while the surface of large microparticles $(25 \mu \mathrm{m})$ underwent degradation. Nanoparticles $(0.24 \mu \mathrm{m})$ showed substantial aggregation and disruption of their spheroid geometry compared to the larger particles. Particles $25 \mu \mathrm{m}, 6 \mu \mathrm{m}$, and $0.24 \mu \mathrm{m}$ in size showed a mass loss of $12 \%, 22 \%$, and $24 \%$ over 10 days, respectively.

To incorporate particles in the EBs we deposited $30000 \mathrm{hESCs}$ and different concentrations of particles $(0.15$ and $0.06 \mathrm{mg}$ of particles per $\mathrm{mL}$ of differentiation medium) in a 96 well plate and forced their aggregation by centrifugation (Fig.1B). At day 2, the diameters of the EBs incorporating different particle sizes were not statistically different $(n=13, P>0.05)$ from those made without particles (Fig. 1C). EBs were transferred at day 2 to a 24 well-plate in order to remove the particles that were not incorporated (around 20-30\%; Fig. 1B). Next, we evaluated the distribution of particles incorporated in the EBs. Particles were distributed across the constructs with higher frequency in the center (Fig. 1D). Particles $6 \mu \mathrm{m}$ and $0.24 \mu \mathrm{m}$ in size showed aggregation in EBs, but $20 \mu \mathrm{m}$ particles did not.
The cytotoxicity of PLGA particles incorporated in EBs was assessed by a Live-Dead assay after 2 days of EB formation. Cell viability was above $80 \%$, and no significant differences were observed among the different conditions tested (Supporting Information Fig. S2). As assessed by an MTT assay at day 2, the metabolic activity in EBs with particles was not statistically significant $(n=6, P>0.05)$ to the one observed in EBs without particles (Fig. 1E). Moreover, no statistically significant increase $(n=6, P>0.05)$ in cellular metabolic activity was observed over 10 days (Fig. 1E), suggesting that EBs were in a non-proliferative state, conducive to differentiation. Taken together, our results indicate that PLGA particles had little effect on the viability of EBs.

Previous studies have shown that particles up to $1 \mu \mathrm{m}$ can be internalized by receptor-mediated endocytosis by some cell types. ${ }^{[11-14]}$ Particle uptake by hESCs at different stages of differentiation is largely unknown, as is their final cellular localization. We studied cellular uptake of fluorescent-labeled PLGA particles of different sizes by fluorescence-activated cell sorting (FACS; Fig. 2A). For this purpose, EBs with or without tetramethylrhodamine-5-(and-6)-isothiocyanate (TRITC)-labelled
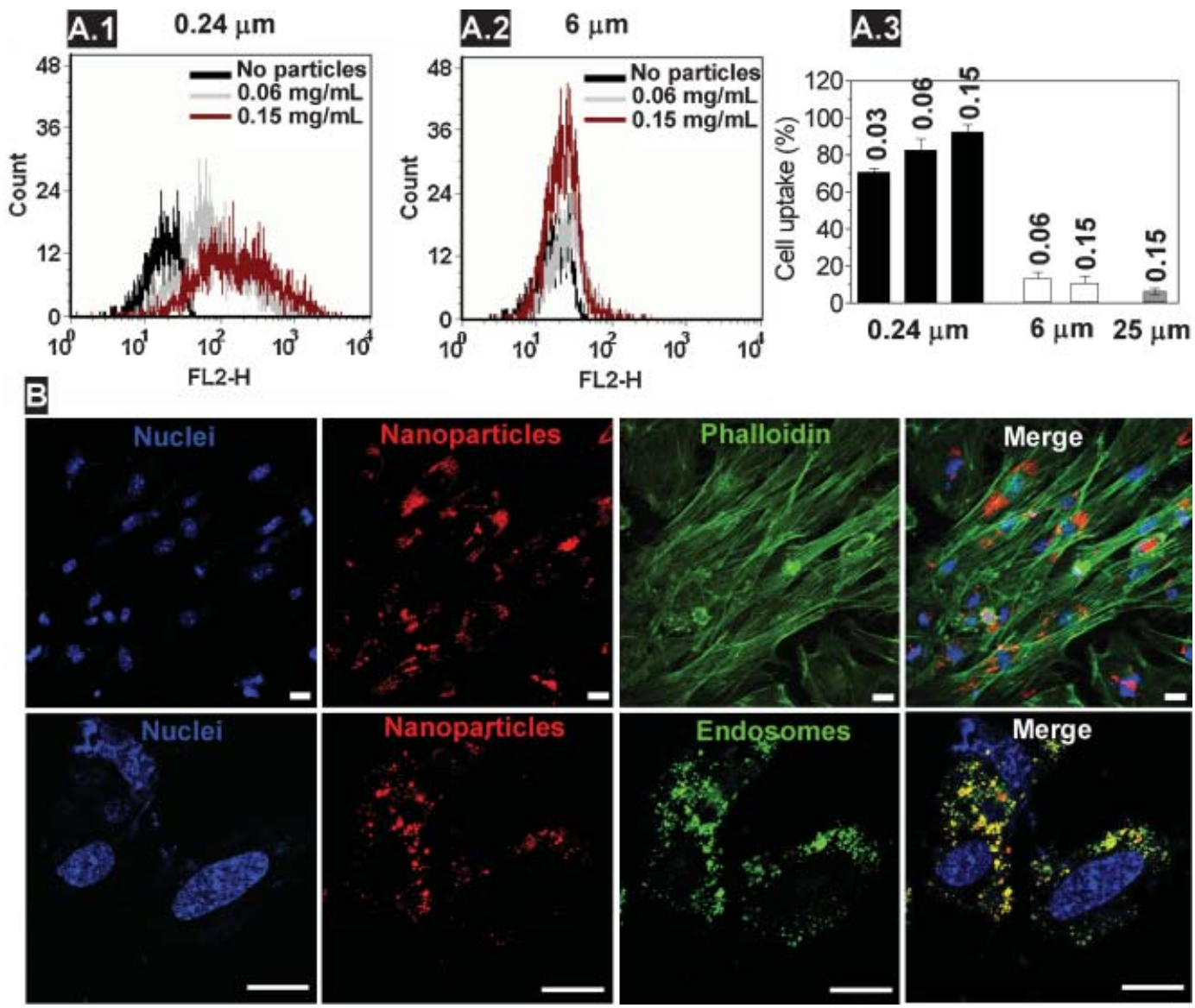

Figure 2. Cellular uptake of particles. A) Flow cytometry analyzes of particle uptake. Quantification of TRITC-positive cells indicated that minimal cellular uptake of particles is observed for particles 6 and $25 \mu \mathrm{m}$ in size, while substantial cellular uptake is observed for nanoparticles. B) Confocal microscopy of cellular uptake of nanoparticles EBs were formed by the aggregation of $30000 \mathrm{hESCs}$ and $0.15 \mathrm{mg} \mathrm{mL}^{-1}$ of particles. Blue Topro-3 stains the nucleus, green lysosensor indicates endosomes, green phalloidin indicates cytoplasm, and TRITC-labelled nanoparticles are displayed in red. Nanoparticles can be seen co-localized with endosomes as a yellow color and distributed mainly in the perinuclear region. For all pictures, the scale bar corresponds to $20 \mu \mathrm{m}$. 
particles at concentrations between $0.03 \mathrm{mg} \mathrm{mL}^{-1}$ and $0.15 \mathrm{mg} \mathrm{mL}^{-1}$ were dissociated at day 2 , the cells plated in a 24 well plate for $18 \mathrm{~h}$, washed to remove loosely bound particles, and characterized by FACS. Nanoparticles $(0.24 \mu \mathrm{m}$ in size) were highly taken up by cells. Increasing the initial dose of nanoparticles in EBs resulted in a higher mean fluorescence intensity as well as an increased fraction of the cell population associated with the fluorescence, indicative of particles with the cells (Fig. 2A). At the highest dose of $0.15 \mathrm{mg} \mathrm{mL}^{-1}$, over $90 \%$ of the population was positive for the nanoparticles. Our FACS results further show that 6 and $25 \mu \mathrm{m}$ microparticles were rarely taken up by cells since only $13 \%$ of the cell population was positive for the microparticles.

Confocal microscopy was performed to confirm that PLGA nanoparticles were located intracellularly, instead of adsorbing onto the cell surface and to identify the exact intracellular location following internalization. For this purpose, EBs incorporating fluorescent-labeled particles at a concentration of $0.15 \mathrm{mg} \mathrm{mL}^{-1}$ of differentiation medium were dissociated at day 2 , the cells plated in a 24 well plate for $18 \mathrm{~h}$, fixed, and then labeled either with phalloidin to stain cellular actin or lysoSensor green to stain endosomes (Fig. 2B). Images of hESCs reconstructed from $\mathrm{z}$-stacks of confocal images indicated extensive cellular uptake of nanoparticles (Fig. 2B) and low uptake of microparticles (Supporting Information Fig. S3). Particles taken up by hESCs were in general localized in the perinuclear region. This agrees with other reports showing perinuclear accumulation of nanomaterials in primary cells ${ }^{[14]}$ or mesenchymal stem cells. ${ }^{[15]}$ Furthermore, our results show that most of the particles were localized in endosomes as shown by the co-localization of the particles with the lysoSensor green staining. We further characterized the cellular uptake of nanoparticles in EBs by transmission electron microscopy (TEM) analyses. TEM results confirm that nanoparticles are taken up by hESCs in EBs (Supporting Information Fig. S4).

Regulatory factors that are known to contribute to early vascular development include vascular endothelial growth factor $\left(\mathrm{VEGF}_{165}\right),{ }^{[16]}$ basic fibroblast growth factor (bFGF), ${ }^{[17]}$ and placenta growth factor $(\mathrm{PlGF}) .{ }^{[18]} \mathrm{VEGF}_{165}$ mediates its responses primarily by activating Flt-1 (also called VEGF-R1) and Flk-1/KDR (also called VEGF-R2). ${ }^{[19]}$ PlGF and bFGF mediate their effects through Flt-1 and FGF receptor tyrosine kinases, respectively. ${ }^{[19]}$ The three receptors are expressed in EBs at early stages of differentiation and in some cases in undifferentiated hESCs. ${ }^{[2,20]}$ Since these receptors are localized at the surface of cells, growth factor release should be extracellular. Therefore, microparticles of $6 \mu \mathrm{m}$ in size were selected for subsequent experiments. These particles are minimally taken up by cells compared to nanoparticles, and they are more highly dispersed in composite EBs than $25 \mu \mathrm{m}$ particles and may therefore produce a more homogeneous differentiation. We characterized the release kinetics of PLGA particles loaded with growth factors (Fig. 3A). Quantitative differences in the cumulative mass of growth factor released from the particle preparations were observed (Fig. 3A and Supporting Information Table S2). The release kinetics of all three growth
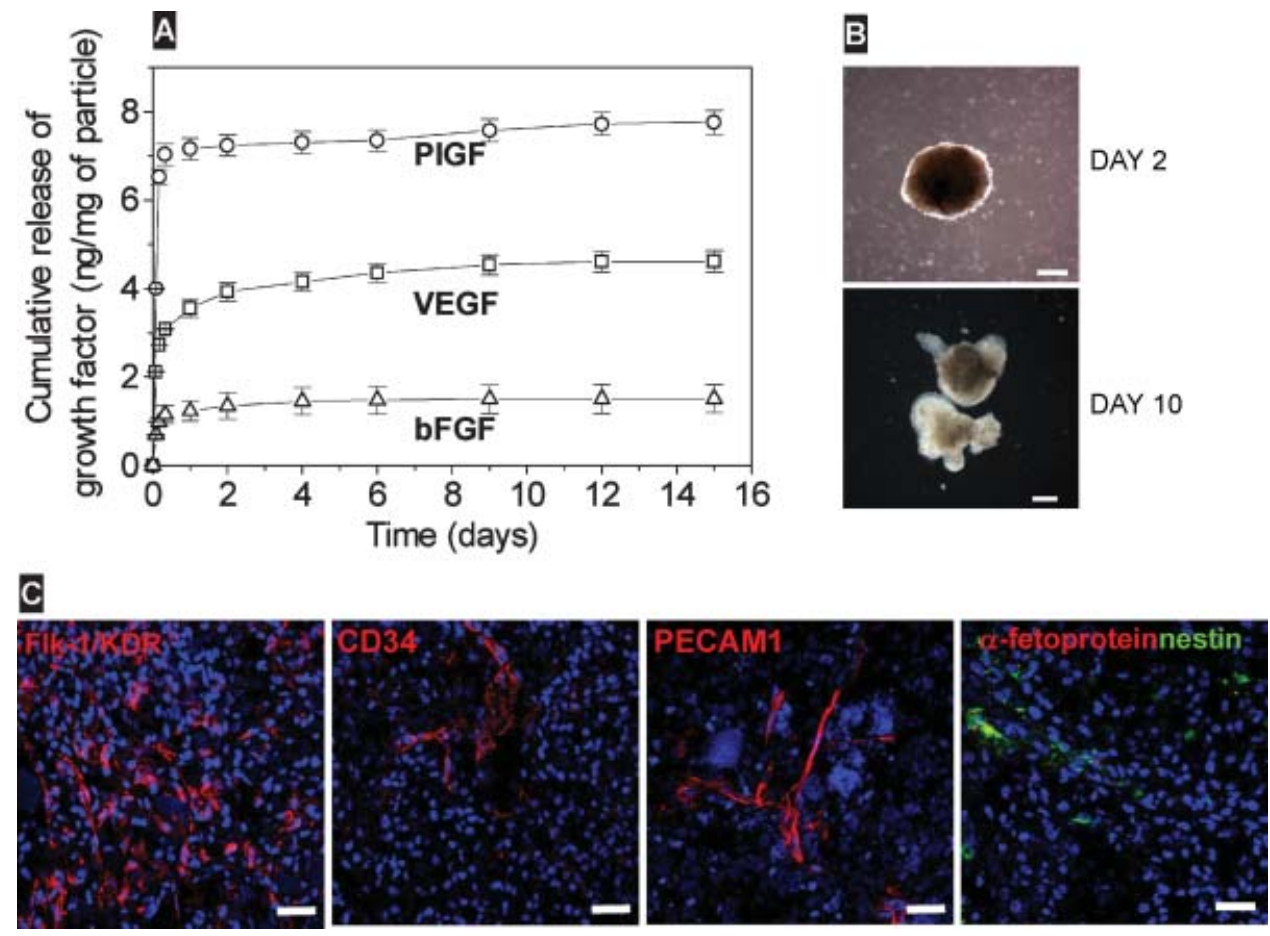

Figure 3. Growth-factor release in microparticles and protein expression in EBs containing growth factor-releasing microparticles. A) Release profile of VEGF $_{165}$, PIGF and bFGF. B) EBs at day 2 and 10 containing PIGF-releasing microparticles. Bar corresponds to $200 \mu \mathrm{m}$. C) Localization and organization of endothelial (Flk-1, CD34 and PECAM1) markers in EBs containing PIGF-releasing microparticles, as evaluated by confocal microscopy. Nestin/ $\alpha$-fetoprotein co-staining was also performed to assess the differentiation of EBs into the ectoderm and endoderm germ layers. Bar corresponds to $50 \mu \mathrm{m}$. 
factor-loaded microparticles showed an initial burst followed by a slow or negligible release. After 10 days, $1 \mathrm{mg}$ of particles had released 7.6, 4.5, and 1.5 ng of PIGF, VEGF and bGFG, respectively, corresponding to the release of $20 \%, 10 \%$, and $5 \%$ of the initial amount of encapsulated growth factor. Previously, we have shown that VEGF released from this particle formulation was functional, as assessed by a cell survival assay. ${ }^{[21]}$

We then incorporated these growth-factor-containing particles in EBs for 10 days (Fig. 3B) and characterized the vascular differentiation of the EBs at the protein and gene levels, compared to constructs containing blank particles. For each EB we used $25 \mu \mathrm{g}$ of microparticles, which corresponds to approximately 195000 particles as measured with a hemocytometer (see Experimental). Since roughly $70 \%$ of the particles initially used in each well were incorporated in the EBs, this corresponds to approximately 136500 particles or a mass of $17.5 \mu \mathrm{g}$ per EB. The genes and proteins analyzed included endothelial cell adhesion molecules such as PECAM1, vascular endothelial-cadherin (VE-cad) and CD34; growth factor receptors such as vascular endothelial growth factor receptor-2 (Flk-1/ KDR) and Tie-2; endothelial glycoproteins such as von Willebrand factor (vWF) and secreted endothelial molecules including angiopoietin 2 (Ang2) ${ }^{[3]}$ Immunofluorescence studies showed that of the three endothelial cell markers assessed
(PECAM1, CD34, and Flk-1/KDR), Flk-1/KDR was the highly expressed in all the conditions tested, particularly in EBs containing VEGF- releasing particles (approximately $40-50 \%$ of the cells displayed this marker; Fig. 3C and Supporting Information Fig. S4). Below $10 \%$ of cells in EBs expressed PECAM1 and CD34 markers.

Figure $3 \mathrm{C}$ shows the localization and organization of endothelial markers in EBs containing PIGF particles. CD $34^{+}$and PECAM1 ${ }^{+}$cells seem to organize in vascular networks, as previously reported by us in regular EBs, ${ }^{[2,21]}$ while $\mathrm{KDR} / \mathrm{Flk}-1^{+}$cells are randomly organized. Similar results were obtained for the remaining composite EBs (Supporting Information Fig. S4).

We then evaluated at the gene level the differentiation of EBs. VEGF, PlGF, and bFGF microparticles improved the vascular differentiation of EBs, as $3-5$ of the 7 genes are up-regulated as compared to EBs containing blank particles (Fig. 4A). PlGF seems to have a greater effect than the other two growth factors under the conditions tested. We then compared the gene expression of EBs-containing growthfactor-releasing particles and EBs formed by a regular approach (regular EBs), that is, formed by the removal of hESC colonies from a feeder layer and exposed to medium supplemented with each of growth factors $\left(50 \mathrm{ng} \mathrm{mL}^{-1}\right)$ for 10 days (Fig. 4B). The vascular differentiation effect of VEGF in these regular EBs was higher than the one observed in EBs
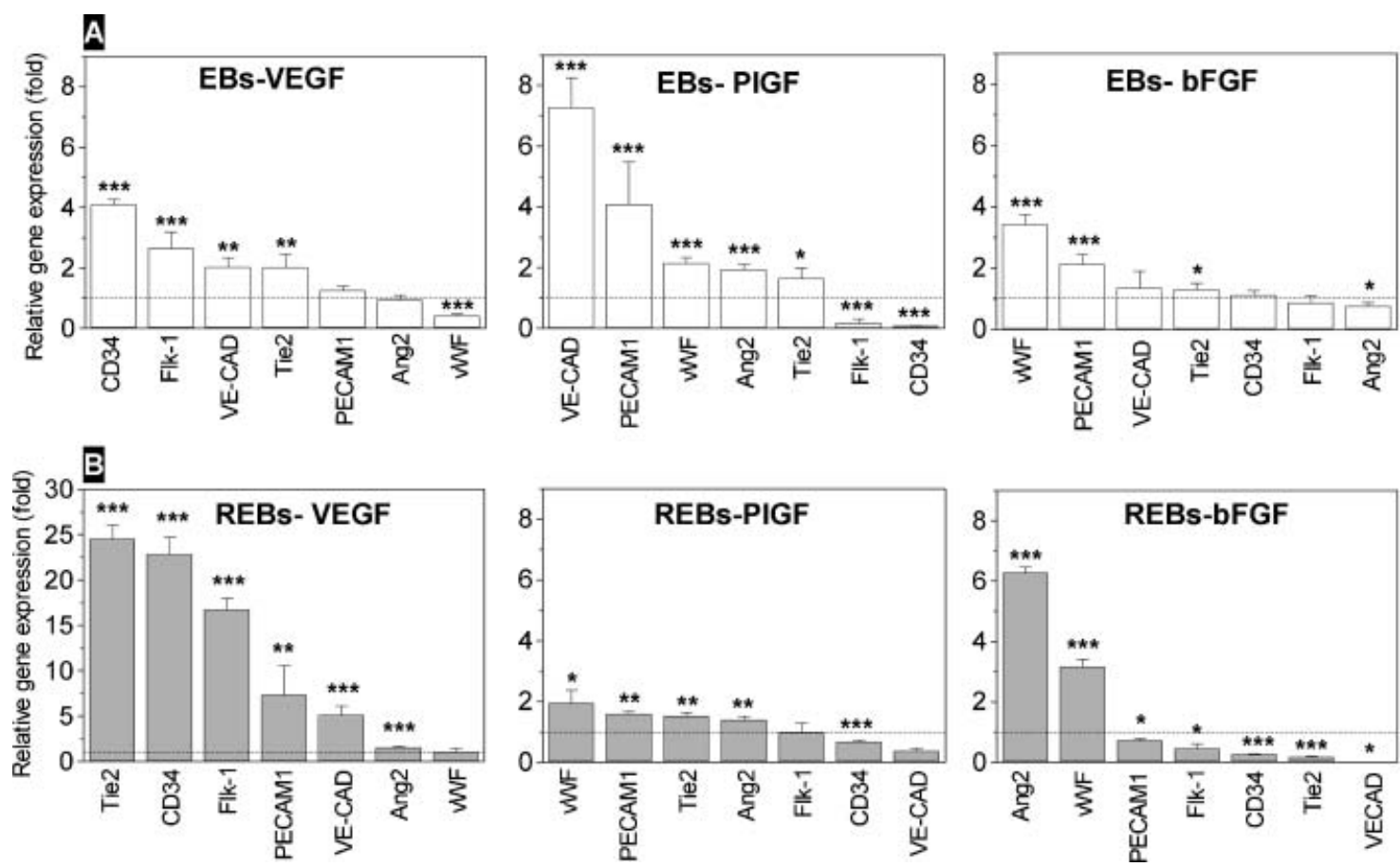

Figure 4. Endothelial differentiation on regular EBs or EBs containing growth factor-releasing microparticles. Endothelial gene expression in EBs containing growth factor- releasing microparticles (A; EBs) and regular EBs (B; REBs) as assessed by quantitative RT-PCR. REBs were formed by removing colonies of undifferentiated hESCs from the feeder layer and let them in suspension. EBs containing growth factor-releasing microparticles were formed by the forced aggregation of single hESCs and microparticles. In both cases, RNA was isolated at day 10 during differentiation and subjected to RT-PCR analysis. Quantification of target genes was performed relatively to the reference GAPDH gene, and normalized to the expression of the different markers in EBs with blank particles or REBs cultured without growth factors. *, **, and *** denote statistical significance $(P<0.05, P<0.01$, and $P<0.001$, respectively). 
containing VEGF microparticles. Surprisingly, although EBs containing bFGF or PlGF microparticles were exposed to lower concentrations of growth factors than EBs exposed to exogenous factors (for bFGF approx. $175 \mathrm{pg} \mathrm{mL}^{-1}$ vs. $50 \mathrm{ng} \mathrm{mL}^{-1}$ and for PlGF approx. $887 \mathrm{pg} \mathrm{mL}^{-1}$ vs. $50 \mathrm{ng} \mathrm{mL}^{-1}$ ) the vascular differentiation was comparable or even superior. For example, the expression of PECAM-1, a definitive marker for endothelial cells, ${ }^{[2,17]}$ was two or four-fold higher in EBs containing bFGF or PlGF microparticles than regular EBs exposed to those growth factors, respectively. Therefore our results show that the release of small concentrations of growth factors by microparticles within EBs contributed largely to their vascular differentiation.

We assessed whether the growth-factor-releasing particles in EBs could limit their differentiation toward other germ layers (i.e., ectoderm and endoderm germ layers). For that purpose we evaluated the expression of genes and proteins related to neuronal differentiation (ectodermal differentiation) including nestin and neurofilament $68 \mathrm{Kd},{ }^{[4,7,21]}$ and markers related to hepatic differentiation (endodermal differentiation) including albumin and $\alpha$-fetoprotein. ${ }^{[7,21]}$ PlGF microparticles in EBs substantially reduced the neuronal and hepatic differentiation of cells as compared to EBs containing blank microparticles (Fig. 3C and Supporting Information Fig. S5), and this effect was greater than in regular EBs exposed to PlGF. In addition, bFGF microparticles in EBs substantially reduced the hepatic but not the neuronal differentiation, while VEGF microparticles incorporated in EBs have no effect in the hepatic differentiation and variable effect in the neuronal differentiation (Supporting Information Figs. S4 and S5).

In summary, we report a simple methodology to control the differentiation of hESCs into the vascular lineage. Vascular cells are very promising for tissue engineering applications and the treatment of cardiovascular diseases such as myocardial ischemia. ${ }^{[22]}$ Our approach uses biodegradable and biocompatible components and allows for the control of several variables of potential importance for cell differentiation including growth factor concentration, spatial positioning of growth factor and combinatorial release of bioactive molecules. This platform can likely be extended to the differentiation of hESCs into other cell lineages.

\section{Experimental}

Preparation of Growth-Factor-Releasing Particles: Particles were prepared using a double emulsion solvent evaporation procedure. For nanoparticles, PLGA (100 mg, inherent viscosity $0.16-0.24 \mathrm{dl} \mathrm{g}^{-1}$, Boehringer Mannheim) polymer was dissolved in ethyl acetate $(1 \mathrm{~mL}$, Aldrich) with magnesium hydroxide (4 mg, Aldrich). Then, $50 \mu \mathrm{L}$ of an aqueous solution of BSA $\left(50 \mathrm{mg} \mathrm{mL}^{-1} \mathrm{BSA}\right.$ in $10 \mathrm{~mm}$ phosphate buffer, $\mathrm{pH} 7.4$ containing $5 \mu \mathrm{g}$ of heparin) was added to the organic polymer solution, and the aqueous and organic phases were emulsified by sonication (Vibra Cell, Sonics \& Materials, Inc., Danbury, CT). For microparticles 6 and $25 \mu \mathrm{m}$, PLGA $(100 \mathrm{mg}$, inherent viscosities of $0.16-0.24$ or $0.32-0.44 \mathrm{dl} \mathrm{g}^{-1}$ ) polymer (see Supporting Information Table S1) was dissolved in methylene chloride ( $2 \mathrm{~mL}$, Aldrich) with magnesium hydroxide $(4 \mathrm{mg})$. Then, $50 \mu \mathrm{L}$ of PIGF or bFGF or
$\operatorname{VEGF}_{165}$ (R\&D Systems) $\left(5 \mu \mathrm{g}\right.$; in $50 \mathrm{mg} \mathrm{mL} \mathrm{mL}^{-1} \mathrm{BSA}$ in $10 \mathrm{~mm}$ phosphate buffer, pH 7.4 containing $5 \mu \mathrm{g}$ of heparin) or BSA solution was added to the organic polymer solution, and the aqueous and organic phases were emulsified by sonication (Vibra Cell, Sonics \& Materials, Inc., Danbury, CT). For the preparation of fluorescence-labeled particles, $20 \mu \mathrm{L}$ of a tetramethylrhodamine-5-(and-6)-isothiocyanate (TRITC; Molecular Probes; $10 \mu \mathrm{g} \mathrm{mL}^{-1}$ in ethyl acetate or methylene chloride) was added to the organic phase of each formulation, maintaining the other components. Microparticles and nanoparticles were prepared and characterized as previously described [23] and stored at $-20^{\circ} \mathrm{C}$. The morphology of PLGA particles during growth factor release was evaluated by scanning electron microscopy (SEM). For that purpose, they were mounted onto an aluminum stud, and gold coated by plasma vapor deposition. To determine the density of particles $6 \mu \mathrm{m}, 2.5 \mathrm{mg}$ of microparticles were suspended in $1.25 \mathrm{~mL}$ of PBS after which a small volume of this solution was loaded into a hemocytometer, visualized using a phase contrast microscope $(40 \times)$, and counted. This procedure was repeated for at least two different batches to yield an overall estimate of $7.8 \times 10^{6}$ ( $\pm 9.5 \%$ S.D.) microparticles $\mathrm{mg}^{-1}$ powder.

Growth Factor Release Studies: Microparticles $(10 \mathrm{mg})$ were placed in PBS $(0.5 \mathrm{~mL})$ and incubated under mild agitation, at $37^{\circ} \mathrm{C}$. At specific intervals of time, the particle suspension was centrifuged (at $4,000 \mathrm{rpm}$ for $2 \mathrm{~min}$ ) and $0.4 \mathrm{~mL}$ of the release medium removed and replaced by a new one. The reserved supernatant was stored at $-20^{\circ} \mathrm{C}$ until the growth factor content in release samples was assessed using an enzyme-linked immunosorbent assay (ELISA; R\&D Systems). Concentrations of growth factors were determined by comparison to a standard curve. All analyses were conducted in duplicate. To determine the loading efficiency of PLGA particles, $2.5 \mathrm{mg}$ of microparticles were dissolved in $0.5 \mathrm{~mL}$ of $1 \mathrm{~N} \mathrm{NaOH}$ overnight and the absorbance measured at $284 \mathrm{~nm}$. The results are expressed as a percentage of the ratio of protein encapsulated to total protein used.

hESC Culture and Formation of EBs: Undifferentiating hESCs (H9, passages 25 to 50; WiCell, Wisconsin) were grown on an inactivated mouse embryonic fibroblast (MEF) feeder layer, as previously described [2,21]. To induce the formation of EBs, undifferentiated hESCs were treated with $2 \mathrm{mg} \mathrm{mL}^{-1}$ type IV collagenase for $1-2 \mathrm{~h}$, and then transferred $(2: 1)$ to low attachment plates $(\varnothing=10 \mathrm{~cm}$, Corning) containing $10 \mathrm{~mL}$ of differentiation medium (80\% knockout-Dulbecco's Modified Eagle Medium, supplemented with $20 \%$ fetal bovine serum (FBS, Invitrogen), $0.5 \%$ L-glutamine, $0.2 \% \beta$-mercaptoethanol, and $1 \%$ nonessential amino acids (all from Invitrogen)). The EBs obtained by this procedure are named regular EBs. They were cultured for 10 days at $37^{\circ} \mathrm{C}$ and $5 \%$ $\mathrm{CO}_{2}$, in a humidified atmosphere, with changes of media every 2-3 days. To assess the effect of bFGF, PlGF, and $\mathrm{VEGF}_{165}$ in the differentiation profile of $\mathrm{EBs}$, the differentiation medium was supplemented with $50 \mathrm{ng} \mathrm{mL}^{-1}$ of growth factor.

Preparation of EBs Containing Nano- and Microparticles: Undifferentiated hESCs seeded on MEFS were treated with $2 \mathrm{mg} \mathrm{mL}^{-1}$ type IV collagenase for $2 \mathrm{~h}$, washed in PBS, then treated with cell dissociation solution for 10 minutes and dissociated by gentle pipetting. EB formation was induced by seeding the desired number of hESCs in $168.5 \mu \mathrm{L}$ of differentiation medium containing 0.15 or 0.06 or $0.03 \mathrm{mg} \mathrm{mL}^{-1}$ of particles in each well of 96 well, round-bottomed, low-attachment plates (Nunc, Denmark). The plates were then centrifuged at $1200 \mathrm{~g}$ for 4 minutes to aggregate the cells. At day 2, the formed EBs were removed from the 96 well-plate and placed in a low-adherent 24 well plate (4-6 EBs per well). The differentiation medium was changed every 2-3 days.

Viability and Metabolic Activity of EBs Containing Particles: Cell viability of EBs containing particles was determined using a LIVE/ DEAD kit (Molecular Probes) containing calcein AM $\left(2 \mu \mathrm{g} \mathrm{mL}{ }^{-1}\right.$, in PBS) and ethidium homodimer $\left(4 \mu \mathrm{g} \mathrm{mL}^{-1}\right.$, in PBS). The EBs were placed in the kit solution for 20 min and visualized under a Zeiss LSM 510 confocal microscope. This kit measures the membrane integrity of 
cells. Viable cells fluoresce green through the reaction of calcein AM with intracellular esterase, whereas non-viable cells fluoresce red due to the diffusion of ethidium homodimer across damaged cell membranes and binding with nucleic acids.

The metabolic activity of EBs containing particles was measured through a MTT assay after 2 and 10 days of culture. The MTT solution $\left(0.2 \mathrm{~mL}, 0.45 \mathrm{mg} \mathrm{mL}^{-1}\right.$ in differentiation medium) was added to each well $\left(96\right.$ well plate) containing ca. $6 \mathrm{EBs}$ for $3 \mathrm{~h}$, at $37^{\circ} \mathrm{C}$. After that time, the medium was removed and $0.1 \mathrm{~mL}$ of DMSO was added for $15 \mathrm{~min}$. The absorbance was then measured spectrophotometrically at $540 \mathrm{~nm}$.

FACS Analysis: EBs differentiated for 2 days were treated with trypsin $(0.25 \%$ in PBS) for 5 minutes and dissociated by gentle pipetting. Single cells were plated in a $1 \%$ gelatin-coated 24 well plate $\left(1 \times 10^{5}\right.$ cells well $\left.^{-1}\right)$ containing differentiation medium. After $18 \mathrm{~h}$, the cells were washed with PBS and dissociated with trypsin. The single cell suspensions were then analyzed on a FACScan (Becton Dickison), using a CellQuest software.

Confocal Microscopy Analysis: At day 10 of differentiation, EBs containing particles were transferred to gelatin-coated cover slips with differentiation medium, allowed to attach overnight, and then, fixed with $4 \%(\mathrm{w} / \mathrm{v})$ paraformaldehyde for 30 minutes at room temperature. To evaluate the distribution of TRITC-labelled particles within EBs, the cell nuclei were stained with Topro-3 (Sigma) followed by the examination with a Zeiss LSM 510 confocal microscope.

To evaluate endothelial marker expression in EBs containing growth factor-releasing particles, fixed EBs were blocked with $3 \%$ BSA solution, and the cells stained for $1 \mathrm{~h}$ with the following anti-human primary antibodies: PECAM1 (Dako), CD34 (Dako), Flk-1/KDR (Santa Cruz Biocehmicals), nestin (R\&D Systems) and $\alpha$-fetoprotein (Dako). In each experiment, an isotype-matched IgG control was used. Binding of primary antibodies to specific cells was detected with anti-mouse IgG $\mathrm{Cy} 3$ conjugate, anti-rabbit $\mathrm{IgG} \mathrm{Cy} 3$ conjugate or anti-mouse IgG FITC (all from Sigma). Cell nuclei were stained with Topro- 3 and the slides examined by confocal microscopy.

To evaluate the cellular uptake of particles, EBs containing particles and differentiated for 2 days were dissociated by trypsin $(0.25 \%$ in PBS) and plated $\left(1 \times 10^{5}\right.$ cells/well $)$ in a $1 \%$ gelatin-coated chamber slides containing differentiation medium for $18 \mathrm{~h}$. For lysosensor staining, cells were stained with lysosensor green $(2 \mu \mathrm{M}$ in differentiation medium; Molecular Probes) for $1 \mathrm{~h}$, washed with PBS, and then fixed with $4 \%(\mathrm{w} / \mathrm{v})$ paraformaldehyde for 15 minutes at room temperature. Cell nuclei were stained with Topro-3 (0.002\%, v/v, in PBS) for $30 \mathrm{~min}$. For phalloidin staining, cells were fixed with $4 \%(w / v)$ paraformaldehyde for 15 minutes at room temperature, washed with PBS, permeabilized with $0.1 \%$ Triton X-100 in PBS, washed again with PBS and finally stained with $50 \mu \mathrm{g} \mathrm{mL} \mathrm{m}^{-1}$ FITC-phalloidin (Sigma) for 30 minutes at room temperature. The stained cells were then washed with PBS and cell nuclei stained with Topro-3.

Reverse Transcription-Polymerase Chain Reaction (RT-PCR) Analysis: Total RNA from regular EBs or EBs containing microparticles (3-4 separate experiments and combined in equal amounts) was isolated with RNeasy Minikit (Qiagen, Valencia). cDNA was prepared from $1 \mathrm{mg}$ total RNA using Taqman Reverse transcription reagents (Applied Biosystems, CA). Quantitative PCR (qPCR) was performed using Power SYBR Green PCR Master Mix (Applied Biosystems) and the detection using a 7500 Fast Real-Time PCR System (Applied Biosystems, Foster). Quantification of target genes was performed relatively to the reference GAPDH gene: relative expression $=2^{[-(C t--\mathrm{Ct})]}$ sampleGADPH . The mean minimal cycle threshold values $(C t)$ were calculated from quadruplicate reactions. Then, the relative gene expression for EBs containing growth factor-releasing microparticles was normalized to the relative gene expression found in
EBs containing blank particles. Primer sequences are published as Supporting Information (Supporting Information Table S3).

Statistical Analysis: An unpaired t test or one-way analysis of variance with Bonferroni post test was performed for statistical tests by using GraphPad Prism 4.0 (San Diego, CA). Results were considered significant when $P \leq 0.05$.

Received: September 22, 2007

Revised: December 27, 2007

Published online: May 16, 2008

[1] L. M. Hoffman, M. K. Carpenter, Nat. Biotechnol. 2005, 23, 699.

[2] L. S. Ferreira, S. Gerecht, H. F. Shieh, N. Watson, M. A. Rupnick, S. M. Dallabrida, G. Vunjak-Novakovic, R. Langer, Circ. Res. 2007, 101, 286.

[3] S. Levenberg, J. S. Golub, M. Amit, J. Itskovitz-Eldor, R. Langer, Proc. Natl. Acad. Sci. USA 2002, 99, 4391.

[4] S. C. Zhang, M. Wernig, I. D. Duncan, O. Brustle, J. A. Thomson, Nat. Biotechnol. 2001, 19, 1129.

[5] E. Poon, F. Clermont, M. T. Firpo, R. J. Akhurst, J. Cell Sci. 2006, 119, 759.

[6] C. M. Cameron, W. S. Hu, D. S. Kaufman, Biotechnol. Bioeng. 2006 94, 938.

[7] S. Gerecht-Nir, S. Cohen, J. Itskovitz-Eldor, Biotechnol. Bioeng. 2004, 86, 493.

[8] E. R. Edelman, M. A. Nugent, M. J. Karnovsky, Proc. Natl. Acad. Sci. USA 1993, 90, 1513 .

[9] M. J. Mahoney, W. M. Saltzman, Nat. Biotechnol. 2001, 19, 934.

[10] E. S. Ng, R. P. Davis, L. Azzola, E. G. Stanley, A. G. Elefanty, Blood 2005, 106, 1601

[11] K. Y. Win, S. S. Feng, Biomaterials 2005, 26, 2713.

[12] M. P. Desai, V. Labhasetwar, E. Walter, R. J. Levy, G. L. Amidon, Pharm. Res. 1997, 14, 1568.

[13] B. D. Chithrani, A. A. Ghazani, W. C. Chan, Nano Lett. 2006, 6, 662

[14] L. Lacerda, G. Pastorin, D. Gathercole, J. Buddle, M. Prato, A. Bianco, K. Kostarelos, Adv. Mater. 2007, 19, 1480.

[15] O. Seleverstov, O. Zabirnyk, M. Zscharnack, L. Bulavina, M. Nowicki, J. M. Heinrich, M. Yezhelyev, F. Emmrich, R. O'Regan, A. Bader, Nano Lett. 2006, 6, 2826.

[16] P. Carmeliet, V. Ferreira, G. Breier, S. Pollefeyt, L. Kieckens, M. Gertsenstein, M. Fahrig, A. Vandenhoeck, K. Harpal, C. Eberhardt, C. Declercq, J. Pawling, L. Moons, D. Collen, W. Risau, A. Nagy, Nature 1996, 380, 435.

[17] D. Vittet, M. H. Prandini, R. Berthier, A. Schweitzer, H. MartinSisteron, G. Uzan, E. Dejana, Blood 1996, 88, 3424.

[18] P. Carmeliet, L. Moons, A. Luttun, V. Vincenti, V. Compernolle, M. De Mol, Y. Wu, F. Bono, L. Devy, H. Beck, D. Scholz, T. Acker, T. DiPalma, M. Dewerchin, A. Noel, I. Stalmans, A. Barra, S. Blacher, T. Vandendriessche, A. Ponten, U. Eriksson, K. H. Plate, J. M. Foidart, W. Schaper, D. S. Charnock-Jones, D. J. Hicklin, J. M. Herbert, D. Collen, M. G. Persico, Nat. Med. 2001, 7, 575 .

[19] H. Roy, S. Bhardwaj, S. Yla-Herttuala, FEBS Lett. 2006, 580, 2879.

[20] M. Schuldiner, O. Yanuka, J. Itskovitz-Eldor, D. A. Melton, N. Benvenisty, Proc. Natl. Acad. Sci. USA 2000, 97, 11307.

[21] L. S. Ferreira, S. Gerecht, J. Fuller, H. F. Shieh, G. Vunjak-Novakovic, R. Langer, Biomaterials 2007, 28, 2706.

[22] E. N. Olson, Nat. Med. 2004, 10, 467.

[23] B. A. Pfeifer, J. A. Burdick, R. Langer, Biomaterials 2005, 26, 117. 